27. RAWASHDEH, Y.F.; JORGENSEN, T.M.; OLSEN, L.H. y cols.: "The outcome of detrusor myotomy in children with neurogenic bladder dysfunction". J. Urol., 171: 2654, 2004.

Casos Clínicos

Arch. Esp. Urol., 60, 5 (589-593), 2007

\section{ANEURISMA CIRSOIDE RENAL: UNA RARA CAUSA DE HEMATURIA SEVERA.}

José Gregorio Pereira Arias, Vicente Ullate Jaime, Esther Pereda Martínez', José María Gutiérrez Díez, Ricardo Ateca Díaz-Obregón, Maria Mar Ramírez Rodríguez’ y José Ramón Berreteaga Gallastegui.

Servicio de Urología. Sección de Anatomía Patológica'. Hospital de San Eloy. Baracaldo. Vizcaya. España.

Resumen.- OBJETIVO: Las fístulas arteriovenosas congénitas renales (aneurisma cirsoide) son una realidad clínica excepcional. Aunque con frecuencia son asintomáticas, su presentación como hematuria grave suscita un excelente ejercicio diagnóstico, y a menudo una actuación terapéutica inmediata.

MÉTODOS Y RESULTADOS: Presentamos una paciente de 75 años de edad con un cuadro de hematuria severa anemizante, hipertensión arterial e insuficiencia cardiaca con- gestiva. El diagnóstico de imagen revelo una ureterohidronefrosis renal derecha con bloqueo vesical por coágulos. El estudio endoscópico (cistoscopia y ureterorenoscopia) alertó sobre el origen renal derecho de la hemorragia, precisando finalmente una nefrectomía resolutiva. El diagnóstico histológico objetivó la presencia de una formación redondeada de múltiples canales vasculares arteriales y venosos en la submucosa pielocalicial con erosión focal epitelial, compatible con fístula arteriovenosa congénita renal (aneurisma cirsoide). Revisamos aspectos diagnósticos y terapéuticos en la literatura.

CONCLUSIONES: Las fístulas arteriovenosas congénitas renales representan un dilema diagnóstico. Pueden presentarse de forma asintomática o bien condicionar clínica derivada del shunt y alto gasto cardiaco (cardiopatía hipertensiva e insuficiencia cardiaca congestiva) o por erosión e infiltración hemorrágica aguda de la vía urinaria (hematuria renal severa). El tratamiento debe ser conservador mediante la embolización o esclerosis supraselectiva. No obstante, ante fístulas voluminosas, repermeabilización postembolización o en situaciones de inestabilidad hemodinámica, la nefrectomía es una excelente alternativa.

Palabras clave: Fístula arteriovenosa rena. Aneurisma cirsoide renal.

Summary.- OBJECTIVE: Congenital arteriovenous fistulas are an exceptional clinical feature. Although they are frequently asymptomatic, their presentation as severe hematuria pose an excellent diagnostic exercise and often immediate therapeutic action.

METHODS/RESULTS: We report the case of a 75-year-old female patient presenting with severe hematuria producing anaemia, high blood pressure and congestive heart failure. Image tests revealed right ureteral-hydronephrosis with bladder blockage by blood clots. The endoscopic study (cystoscopy and ureterorenoscopy) alerted about the origin of the hematuria from the right kidney, finally requiring nephrectomy as definitive treatment. Pathology revealed the presence of a round formation with multiple vascular channels, arterial and venous, in the pyelocalicial submucosa, with focal epithelial erosion, compatible with congenital arteriovenous fistula. We review the diagnostic and therapeutic features in the literature.

CONCLUSIONS: Renal congenital arteriovenous fistulas represent a diagnostic dilemma. They may present asymptomatic or condition clinical features derived from the shunt and high cardiac output (hypertensive cardiopathy and congestive heart failure) or from the erosion and acute hemorrhage into the urinary tract (severe renal hematuria). Treatment should be conservative with embolization or supraselective 
sclerosis. Nevertheless, in cases of big fistulas, post embolization revascularization, or hemodynamic instability nephrectomy is an excellent option.

Keywords: Renal arteriovenous fistula. Renal cirsoid aneurysm.

\section{INTRODUCCIÓN}

Las malformaciones arteriovenosas en su conjunto son lesiones poco comunes. Se pueden clasificar según sus características celulares ( hemangiomas o malformaciones vasculares), según su flujo vascular (de alto o bajo flujo), según su predominio arterial, venoso, arteriovenoso, linfático o combinado; y según su comportamiento clínico $(1,2)$. Las fístulas arteriovenosas (FAV) a nivel renal pueden ser de origen congénito (22-25\%), idiopáticas (3-5\%) o adquiridas (70-75\%). La FAV (malformaciones vasculares de alto flujo) congénitas, cuando adquieren disposición en nido se denominan aneurismas cirsoides (3). Las FAV adquiridas son más frecuentes y pueden ser secundarias a traumatismos renales (4), tumorales o yatrogénicas como: biopsia renal (5), cirugía percutánea renal, nefrostomía percutánea (6), cirugía abierta y postlitotricia extracorpórea por ondas de choque (7). Su diagnóstico resulta un excelente ejercicio al poder representar la etiología de una hematuria renal grave que precise una actuación inmediata.

\section{CASO CLÍNICO}

Presentamos una paciente de 75 años de edad con antecedentes médicos de histerectomía, colposuspensión

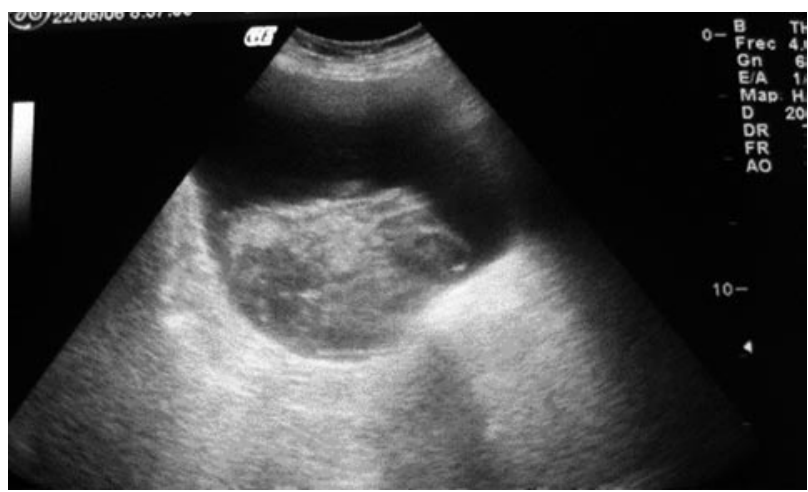

FIGURA 1. Ecografía: lesión nodular vesical endoluminal de $6 \times 6$ cmts, compatible con coagulo vesical.
Marschall-Marchetti y cardiopatía hipertensiva grado II en tratamiento médico. Acude al servicio de urgencias por hematuria macroscópica, disuria, dolor en fosa renal derecha e hipogástiro de varias horas de evolución. El cuadro clínico comenzó 4 días antes con dolor en fosa lumbar derecha asociado a cortejo vegetativo, etiquetado y tratado como posible cólico nefrítico en atención primaria.

En la exploración física se apreciaba moderada palidez mucocutánea, discreto dolor a la palpación en zona lumbar derecha e hipogástrio con aparente globo vesical. La radiografía simple abdominal no determino alteración alguna. El perfil hematológico destacó la presencia de anemia $(3,34$ millones de hematíes, $29,5 \%$ de Hcto y Hemoglobina de $10 \mathrm{gr}$ ) con el resto de parámetros dentro de la normalidad. Se practicó sondaje vesical, objetivando hematuria macroscópica y realizándose lavados vesicales con generosa extracción de coagulos vesicales.

El estudio mediante ultrasonidos informó de la presencia de una discreta ectasia pieloureteral derecha, con riñones de morfología y ecoestructura normal. De igual modo, se objetivo una lesión nodular vesical endoluminal de $6 \times 6 \mathrm{cmts}$, en probable relación con coagulo a dicho nivel (Figura 1). Se procedió al ingreso de la paciente con lavado vesical continuo y enérgico. Se realizó TAC abdominopélvico objetivando una ectasia pieloureteral derecha hasta su porción distal sin evidencia etiológica de la misma. Se apreció retardo en la captación, concentración y eliminación del contraste en la unidad renal derecha sin evidencia de lesiones parenquimatosas. Se insinuó una captación intensa y precoz del contraste en el seno renal derecho con paso rápido de contraste, sin clara significación patológica en ese momento. La vejiga presentaba ocupación endoluminal por caogulos sin alteraciones parietales (Figura 2).

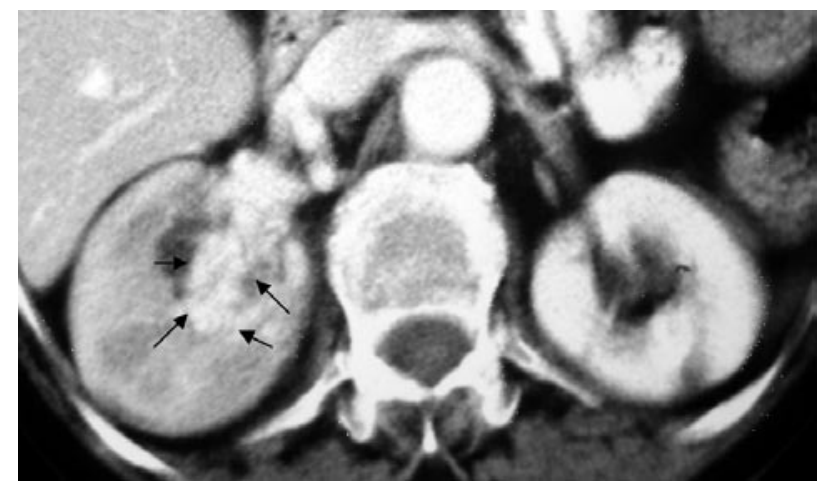

FIGURA 2. TAC abdominopélvico: acumulo de contraste a nivel hiliar con numerosas formaciones arteriales, rápida captación y tránsito del contraste, con retraso en el realce del tejido circundante. 
Antela persistencia y severidad de la hematuria; se realizó exploración endoscópica bajo sedación, observando la presencia de un importante coagulo organizado endovesical, que tras su evacuación evidenció la ausencia de lesiones en la mucosa vesical. No obstante, se apercibió el origen de la hemorragia en la vía urinaria alta derecha, al observar emisión de orina hematúrica por el meato ureteral correspondiente. Se realizó ureterorrenoscopia derecha apreciando una moderada dilatación de la totalidad del uréter. De igual modo se constató la ocupación endoluminal por coagulo pieloureteral e importante hemorragia a nivel piélico sin poder evidenciar la etiología del proceso, dada la intensidad de la misma.

Ante el origen renal de la severa hematuria, la evidente anemización a pesar de la reposición y transfusión (Hcto $21 \%$ y Hemoglobina $7 \mathrm{~g} / \mathrm{dl}$ ) y la ausencia de radiología intervensionista en el centro, se decidió realizar nefrectomía simple derecha permitiendo la precoz resolución del cuadro. El estudio histológico del espécimen revelo la presencia de un múltiples canales vasculares complejos de pared lisa y lumen dilatado en la submucosa piélica y calicial con abundante material hemático en su interior. Los canales presentban morfología arterial y venosa, con erosiones focales del epitelio e infiltración hemorrágica aguda, con vía urinaria dilatada y ocupada por coagulos (Figuras 3 y 4). El diagnóstico definitivo fue de malformación fistulosa arteriovenosa congénita en pelvis renal, compatible con aneurisma cirsoide.

\section{DISCUSIÓN}

El primer sistema funcionante en el embrión es el circulatorio, debido a la imperiosa necesidad de oxígeno para asistir al rápido crecimiento tisular que acontece

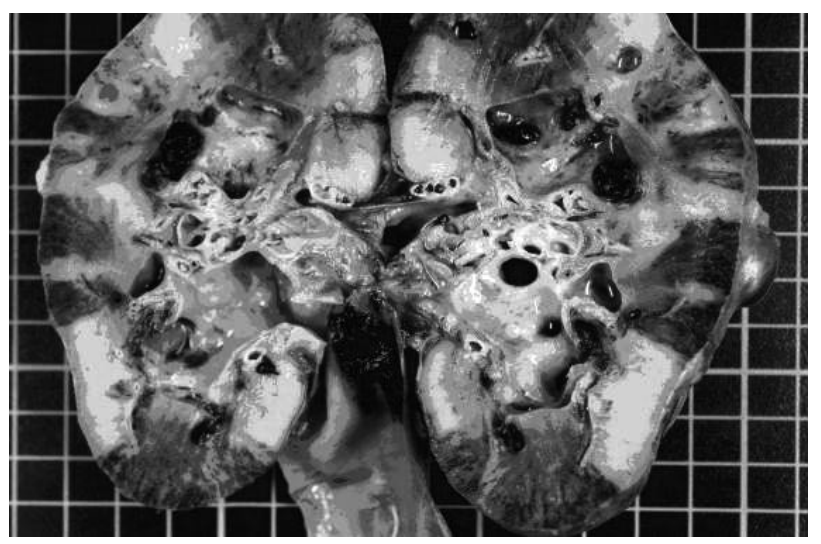

FIGURA 3. Sección sagital del riñón. En la imagen se aprecia uréter dilatado, ocupado por material hemático coagulado. Pelvis renal con proliferación vascular compleja. en este periodo vital. La angiogénesis tiene su origen en el mesodermo y comienza a las tres semanas de gestación. Las malformaciones arteriovenosas congénitas parecen tener su inicio en alteraciones genéticas que distorsionan la habilidad celular para dirigir y regular el entorno periendotelial durante la angiogénesis (8). Estas alteraciones suelen estar presentes en el momento del nacimiento y suelen manifestarse en la infancia-adolescencia cuando adquieren suficiente tamaño para expresarse clínicamente. De igual modo, pueden asociarse a complejos síndromes como: Struge-Weber, Klippel-Trenaunay, Parks-Weber, Cobb, Wybun-Mason, Gorhamstout y otros.

Desde el punto de vista epidemiológico, las FAV congénitas se presentan por igual en ambos sexos, aunque con cierto predomino en el sexo femenino. Pueden afectar por igual a ambas unidades renales con cierto predominio en lado derecho, habiéndose descrito un caso de implicación bilateral (9). Es más habitual, su presentación en la mediana edad (50-60 años), aunque no es infrecuente su debut en la infancia e incluso durante la gestación (10).

Su aparición clínica es imprevisible, siendo asintomáticas durante largos periodos de tiempo. Las malformaciones arteriovenosas congénitas de alto flujo (fístulas arteriovenosas) suelen reflejar un curso clínico más agresivo debido el efecto compresivo que ejercen en los tejidos circundantes, o bien debido al compromiso en el sistema circulatorio. De modo y manera, que el 50\% de los pacientes pueden mostrar signos de insuficiencia cardiaca congestiva e hipertensión diastólica, como ocurrió en nuestra paciente, si bien es cierto que no se asoció a una fístula arteriovenosa. En un tercio de los pacientes el motivo de presentación de las FAV congénitas es la hematuria macroscópica, con frecuencia de carácter recidivante y severo $(3,6,7,11)$. En ocasiones refieren molestias lumbares inespecíficas $(11)$ o fracaso renal (9).

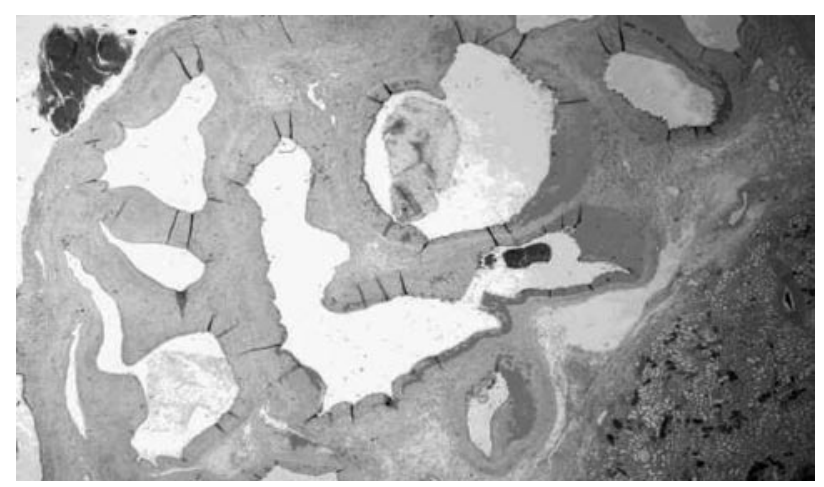

FIGURA 4. Vasos múltiples de morfología arterial y venosa, con luz dilatada, en zona submucosa de la pelvis renal (HE $\times 4$ 4). 
El diagnóstico depende del momento evolutivo (quiescente, expansivo, destructivo y descompensado) de la FAV y suele ser tardío. Se trata, en ocasiones, de un proceso reflexivo de sospecha ante una hematuria de origen incierto, o casual en un estudio de imagen, y con frecuencia de carácter urgente. La exploración física permitiría, ante su sospecha, la auscultación de un soplo abdominal en el $75 \%$ de ocasiones, y con frecuencia el registro de taquicardia basal. La radiología convencional no ofrece ningún signo patognomónico, si bien pueden observarse calcificaciones distróficas en la zona teórica de la FAV a nivel del hilio renal. La urografía endovenosa puede no reflejar anomalía alguna, o bien ofrecer signos dependientes de la situación evolutiva de la FAV. Así, se han documentado imágenes de impronta en el sistema pielocalicial y ocupación endoluminal por coagulos (12).

La Ecografía convencional, aunque se ha descrito un patrón ultrasónico definido por la presencia de lesiones redondeadas anecoicas en el seno renal; pueden no ser suficientes para el diagnóstico. Así, no es infrecuente, que se confunda con hiliosinoquistosis renal o con ectasia pielocalicial (14). Por ello, la mayoría de autores recomienda el empleo del Eco-Doppler para permitir evaluar el carácter vascular de las formaciones anecoicas, y medir su flujo y la presencia de shunt $(13,14)$. La TAC revela la presencia de numerosas formaciones arteriales, con rápida captación y tránsito del contraste al sistema venoso, sin realce del tejido circundante. En nuestra paciente, este detalle pasó desapercibido durante el estudio tomográfico, y fue a posteriori tras filiar el origen renal de la hematuria cuando se sospechó la presencia de una malformación vascular en el hilio renal, sugiriéndose en primera instancia la oportunidad de un hemangioma a dicho nivel. La RNM ofrece múltiples arterias hipertrofiadas con espacios vasculares aumentados conectados por shunts lineales o focales, con baja señal en $\mathrm{T} 1$ y $\mathrm{T} 2$, sin evidencia de masa identificable. No obstante, ambas exploraciones presentan elevada sensibilidad pero baja especificidad; siendo preciso la realización de angiografía, angioTAC o angioresonancia ante la duda diagnóstica (12).

El tratamiento debe perseguir la conservación de la unidad renal implicada, siempre que no exista una situación de inestabilidad hemodinámica. La angiografía permitiría confirmar su diagnóstico, y al mismo tiempo plantear una embolización de la FAV, que de no resultar eficaz, la cirugía aparecería en el escenario terapéutico. Se ha descrito la resolución espontánea de una FAV (15), no obstante se trata de algo excepcional. Ante el hallazgo casual de FAV y en ausencia de hemorragia, fracaso cardiáco, dolor o signos de lesión o erosión local, se podría adoptar una actitud expectante y seguimiento periódico.

La decisión de un abordaje percutáneo y embolización de la FAV, depende de múltiples factores como: preferencias del paciente, estabilidad hemodinámica y dis- ponibilidad de radiología intervensionista en el centro. No obstante, la embolización es la técnica referida por la mayoría de autores. Estas técnicas reflejan un éxito del $68-100 \%$ de los casos, y es más efectiva en las malformaciones congénitas, de escaso volumen y con morfología en nido. Requieren la oclusión completa de la FAV, ya que de no ser así, la recanalización no es infrecuente. Las FAV de alto flujo resultan difíciles de resolver dado la existencia de múltiples arterias y venas, el riesgo de oclusión de arterias con responsabilidad funcional y el escaso tiempo de contacto con el endotelio de los agentes esclerosantes empleados $(4,5,7,16)$. A pesar del elevado éxito de la embolización de las FAV (40\% de curación, $28 \%$ de remisiones parciales), han sido referidas complicaciones en el $52 \%$ de los casos. Se han referido complicaciones como: fracaso renal, necrosis tisular, trombosis venosa, embolismo pulmonar, taquicardia, fiebre y recanalización. De igual modo se ha reportado hemorragia severa por aparición de un shunt arteriopiélico durante el tratamiento mediante embolización de las FAV.

Ante el fracaso de la embolización, la persistencia de hematuria-hemorragia se debe recurrir a la cirugía. El objetivo debe ser conservador, en la medida de lo posible. Así, se han descrito nefrectomías parciales, heminefrectomía, reparación extracorpórea y autotrasplante (17) en pacientes monorrenos, ligadura vascular (12) e incluso abordaje laparoscópico (18). De igual modo, se han ensayado abordajes conservadores como el empleo de la radiofrecuencia (19). No obstante, ante FAV de tamaño importante (> 35-40 mm), lesión unilateral con riñón contralateral funcionante, fracaso de la embolización, persistencia de hemorragia-hematuria de carácter anemizante y fracaso de otras medidas hemostáticas; la nefrectomía sigue siendo vigente y una opción resolutiva, tal y como sucedió con nuestra paciente.

En el escenario histológico, las FAV están constituidas por canales vasculares dilatados con paredes finas de endotelio maduro ( a veces sin membrana elástica interna), con trombos organizados en su luz, nódulos papilares (hiperplasisa endotelial de Masson) o calcificaiones distróficas. Se trata de arterias displásicas con drenaje directo en venas en forma de nidos y sin red capilar $(1,2)$.

A modo de conclusión, las FAV (fístulas arteriovenosas) renales son infrecuentes y deben sospecharse ante la presencia de una hematuria de origen incierto, ante la persistencia de una hematuria tras un traumatismo, biopsia renal o cirugía percutánea renal o ante una insuficiencia cardiaca congestiva e hipertensión arterial inexplicables. El objetivo debe ser conservar el parénquima renal, en la medida de lo posible, mediante embolización-escleroterapia, nefrectomía parcial o cirugía vascular reparadora. En las situaciones de inestabilidad hemodinámica, FAV voluminosas o repermeabilización postembolización, la nefrectomía sigue conservando toda su vigencia 


\section{BIBLIOGRAFÍA y LECTURAS RECOMENDADAS (*lectura de interés $y^{* *}$ lectura fundamental)}

1. BELOV, S.: "Anatomopathological classification of congenital vascular defects". Semin. Vasc, Surg., 6: 219, 1993

**2. GERALD, M.; MANRAJ, K.S.: "Classification, diagnosis, and interventional radiologic management of vascular malformations". Orthopedics Clinc. of North Am., 37: 310, 2006.

3. GARCIA, R.; GONZALEZ, J.; MAGANTO, E.: "Fístula arteriovenosa renal congénita (aneurisma cirsoide)". Urology, 24: 495, 1984.

4. GOMEZ, J.A.; CHICHARRO, J.A.; BONILLA, R.: "Fístula arteriovenosa renal postraumática. Tratamiento conservador mediante embolización supraselectiva". Arch. Esp. Urol., 55: 949, 2002.

5. RIBERA, L.; RODRÍGUEZ, A.; FALCÓ, J.: "Fístula arteriovenosa: complicación de biopsia renal. Embolización supraselectiva". Nefrología, 24: 372, 2004.

6. FERNÁNDEZ, T.; GUZMÁN, P.L.; HITA, G.: "Hematuria masiva diferida baja por fístula arteriovenosa tras nefrostomía percutánea". Arch. Esp. Urol., 56: 1158, 2003.

*7. MARQUEZ, A.J.; DIAZ, G.; RUIZ, J.: "Pseudoaneurisma y fístula arteriovenosa renal: estudio mediante doppler color de quiste simple postlitotricia”. Arch. Esp. Urol., 57: 554, 2004.

*8. TILLE, J.C.; PEPPER, M.S.: "Hereditary vascular anomalies: new insights into their pathogenesis". Arterioscler. Thromb. Vasc. Biol., 24: 1578, 2004.

9. RIEDLINGER, W.F.: "Congenital bilateral renal arteriovenous malformation: an unrecognized cause of renal failure". Pediatr. Dev. Pathol., 7: 285, 2004.

10. McMILLAN, R.D.; ROBINETTE, M.A.: "Congenital arteriovenous malformation of kidney in pregnancy". Urology, 26: 441, 1985.

*11. GUILLEN, M.; LLOPIS, B.; ESTEVE, J.: "Fistula arteriovenosa renal congénita". Arch. Esp. Urol., 34: 91, 1981.

12. MELVIN, E.; SOUGLAS, F.A.: "Congenital renal arteriovenous malformation. Angiography in its diagnosis". Urology, 5: 282, 1975.

*14. NAGANUMA, H.; ISHIDA, H.; KONNO, K.: "Renal arteriovenous malformation: sonographic findings". Abdominal Imaging, 26: 661, 2001.

*15. MISHAL, J.; LEIBOVICI, O.; BREGMAN, L.: "Huge renal arteriovenous malformation mimicking a simple para-pelvic cyst". Urologia Internationalis, 66: 49, 2001.

16. KUBOTA, H.; SAKAGAMI, H.; KUBOTA, Y.: "Spontaneous disappearance of a renal arteriovenous malformation”. Int. J. Urol., 10: 547, 2003.

*17. TROCCIOLA, S.M.; CHAER, R.A.; LIN, S.C.: "Embolization of renal artery aneurysm and arteriovenous fistula. A case report". Vasc. Endovascular Surg., 39: 525,2005

18. GROSS, C.; BRUCKE, P.: "Autotransplantation and extracorporeal repair of a renal vascular abnormality in a patient with a single kidney". Vasa, 17: 225, 1988.

19. SPALIVIERO, M.; NOVICK, A.C.; GILL, I.S.: "Laparoscopic nephrectomy for large renal arteriovenous malformation". Urology, 63: 1176, 2004.

*20. BROWN, D.B.; BRANDES, S.B.: "Radiofrequency ablation of a recanalized renal arteriovenous malformation”. J. Vasc. Interv. Radiol., 16: 403, 2005.
Casos Clínicos

Arch. Esp. Urol., 60, 5 (593-595), 2007

\section{NEVUS AZUL PROSTÁTICO. APORTACIÓN DE UN NUEVO CASO.}

\author{
Pablo Garrido Abad, Milagros Jiménez Gálvez, \\ Manuel Fernández Arjona, Luis Miguel Herranz \\ Fernández, Gloria Bocardo Fajardo, Lorenzo Herrero \\ Torres e lgnacio Pereira Sanz.
}

Servicio de Urología del Hospital Universitario La Princesa. Madrid. España.

Resumen.- OBJETIVO: Poner de manifiesto la escasa incidencia de esta patología prostática y la confusión relativa a la terminología aplicada a la presencia de pigmentos melánicos intraprostáticos.

METODOS/RESULTADOS: Paciente de 66 años con valores elevados de PSA al que tras la realización de biopsia prostática se diagnostica de nevus azul prostático.

CONCLUSIONES: Es un hallazgo diagnóstico sin repercusión clínica para el paciente. Se trata de lesiones benignas que no deben ser confundidas en ningún caso con otras similares de características agresivas.

Palabras clave: Nevus azul. Lesiones pigmentarias. Melanina. Próstata.

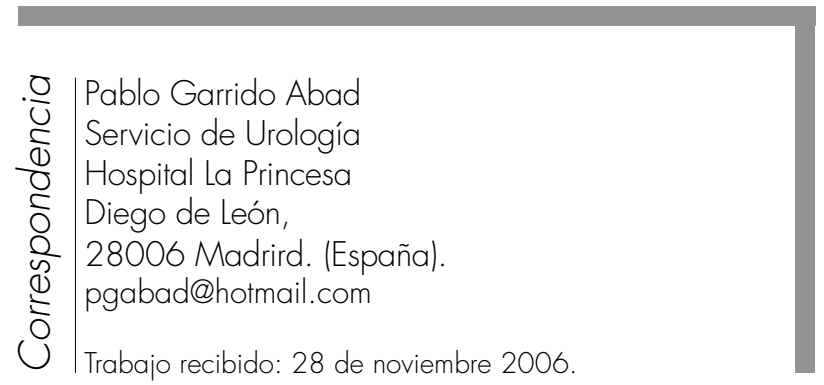

